\title{
Discrete worldline instantons
}

\author{
Christian Schneider, ${ }^{1}$ Greger Torgrimsson, ${ }^{2,3}$ and Ralf Schützhold ${ }^{1,4,5}$ \\ ${ }^{1}$ Fakultät für Physik, Universität Duisburg-Essen, Lotharstrasse 1, 47057 Duisburg, Germany \\ ${ }^{2}$ Theoretisch-Physikalisches Institut, Abbe Center of Photonics, \\ Friedrich-Schiller Universität Jena, Max-Wien-Platz, 1, 07743 Jena, Germany \\ ${ }^{3}$ Helmholtz Institute Jena, Fröbelstieg 3, 07743 Jena, Germany \\ ${ }^{4}$ Helmholtz-Zentrum Dresden-Rossendorf, Bautzner Landstraße 400, 01328 Dresden, Germany \\ ${ }^{5}$ Institut für Theoretische Physik, Technische Universität Dresden, 01062 Dresden, Germany
}

(Received 1 July 2018; published 12 October 2018)

\begin{abstract}
The semiclassical approximation of the worldline path integral is a powerful tool to study nonperturbative electron-positron pair creation in spacetime-dependent background fields. Finding solutions of the classical equations of motion, i.e., worldline instantons, is possible analytically only in special cases, and a numerical treatment is nontrivial as well. We introduce a completely general numerical approach based on an approximate evaluation of the discretized path integral that easily and robustly gives the full semiclassical pair production rate in nontrivial multidimensional fields, and apply it to some example cases.
\end{abstract}

DOI: 10.1103/PhysRevD.98.085009

\section{INTRODUCTION}

A currently unconfirmed prediction of quantum electrodynamics is that of nonperturbative electron-positron pair creation in the presence of a strong electric field [1-3]. Schwinger [4] gave the pair production rate per unit volume $\mathcal{P}_{e^{+} e^{-}}$(or more properly the rate of vacuum decay [5]) in a constant, homogeneous electric field $E$ in $3+1$ dimensions as $(\hbar=c=1)$

$$
\mathcal{P}_{e^{+} e^{-}}=\frac{(q E)^{2}}{4 \pi^{3}} \sum_{n=1}^{\infty} \frac{1}{n^{2}} \exp \left(-n \pi \frac{m^{2}}{q E}\right)
$$

where $q$ is the elementary charge and $m$ the mass of the electrons and positrons. The generalization to inhomogeneous and time dependent background fields is far from straightforward, since this is a nonperturbative effect [as is visible from the inverse dependence on $q$ and $E$ in the exponent of (1)]. Apart from the fundamental interest in this effect as a prototypical example for a nonperturbative phenomenon in quantum field theory, a better understanding is also desirable in view of the various experimental initiatives aimed at reaching ultrahigh field strengths [6].

It is in general difficult to obtain the pair production probability for multidimensional fields. While there has recently been some progress [7-12] in direct numerical

Published by the American Physical Society under the terms of the Creative Commons Attribution 4.0 International license. Further distribution of this work must maintain attribution to the author(s) and the published article's title, journal citation, and DOI. Funded by SCOAP ${ }^{3}$. computation of the exact probability for multidimensional fields, we will instead focus on an approach using the worldline path integral. This formulation is an alternative to path integrals over fields to express amplitudes in quantum field theories. The first steps in this direction were pioneered by Fock, who expressed solutions of the Dirac equation via a four-dimensional Schrödinger-type equation with space and time parametrized by an additional parameter [13]. After Nambu emphasized how beneficial this representation would be in the path integral approach [14], Feynman derived the Klein-Gordon propagator [15] and Dirac propagator [16] in this worldline formulation. In parallel, Schwinger's famous paper [4] used a similar representation. It is possible to approximate this worldline path integral for inhomogeneous fields numerically using discretization and Monte Carlo methods [17-20]. Although our method is based on discretization as well, we use an instanton approach to compute the integrals instead of statistical sampling. This instanton approach is a semiclassical approximation based on a saddlepoint approximation of the worldline path integral where $\mathrm{m}^{2} /(q E)$ plays the role of the large expansion parameter. Both Feynman and Schwinger mentioned the fourdimensional particle's equations of motion in the classical limit, but the first explicit mention of an instanton approximation to the (Euclidean) worldline path integral was given by Affleck, Alvarez and Manton [21]. They derived the pair production rate for a constant homogeneous background field in a way that is very similar to the method used today. The approach was extended to inhomogeneous fields, including the subleading fluctuation prefactor $[22,23]$. An exact analytic treatment is possible in some simple cases [22-24] and analytic approximations allow us to study 
suitable limiting cases [25-27], but in general solutions of the instanton equations of motion have to be found numerically. This can be done using, e.g., shooting methods [28], but the highly nonlinear nature of the equations of motion makes this approach very unstable.

After briefly sketching the semiclassical approximation of the worldline path integral in Sec. II, we introduce a different approach to numerically evaluate the path integral by discretization in Secs. III and IV, and a method to trace families of solutions over a range of field parameters in Sec. V. Finally, we will apply the method to some example cases, both with results known analytically (to assess the accuracy of the approximation) and new examples to demonstrate the scope of the approach in Sec. VI.

\section{WORLDLINE INSTANTON METHOD}

The central object of the method is the effective action $\Gamma_{\mathrm{M}}$, defined using the vacuum persistence amplitude

$$
e^{i \Gamma_{\mathrm{M}}}:=\left\langle 0_{\text {out }} \mid 0_{\text {in }}\right\rangle .
$$

We take the probability for pair creation to be the complement of the vacuum remaining stable, so

$$
P^{e^{+} e^{-}}=1-\left|\left\langle 0_{\text {out }} \mid 0_{\text {in }}\right\rangle\right|^{2}=1-\left|e^{i \Gamma_{\mathrm{M}}}\right|^{2} \approx 2 \Im \Gamma_{\mathrm{M}},
$$

the subscript $\mathrm{M}$ denoting the physical, Minkowskian quantity. We will work with the Euclidean effective action $\Gamma$, related to the Minkowski expression by $\Gamma_{\mathrm{M}}=i \Gamma$, so $\Im \Gamma_{\mathrm{M}}=\Re \Gamma[23]$.

The Euclidean worldline path integral for spinor QED reads (see, e.g., $[29,30])$

$$
\begin{aligned}
\Gamma= & \int_{0}^{\infty} \frac{\mathrm{d} T}{T} e^{-\frac{m^{2}}{2} T} \int_{x(T)=x(0)} \mathcal{D} x(\tau) \\
& \times \Phi[x] \exp \left(-\int_{0}^{T} \mathrm{~d} \tau\left(\frac{\dot{x}^{2}}{2}+i q A(x) \cdot \dot{x}\right)\right),
\end{aligned}
$$

where $A_{\mu}$ is the Euclidean potential representing the external electromagnetic field $F_{\mu \nu}$ and $x_{\mu}(\tau)$ are periodic worldlines in Euclidean space parametrized by the "proper time" $\tau$ with $\dot{x}^{\mu}=\mathrm{d} x^{\mu} / \mathrm{d} \tau$. There exist a couple of different representations of the spin factor, see $[29,31]$. We will use

$$
\Phi[x]=\frac{1}{2} \operatorname{tr} \mathcal{P} e^{\frac{i}{4} \int_{0}^{T} \mathrm{~d} \tau \sigma_{\mu \nu} q F_{\mu \nu}(x),}
$$

with $\mathcal{P}$ denoting path ordering, tr the trace over spinor indices and $\sigma_{\mu \nu}$ the commutator of the Dirac matrices

$$
\sigma_{\mu \nu}=\frac{1}{2}\left[\gamma_{\mu}, \gamma_{\nu}\right]
$$

For certain simple fields [such as a time-independent electrostatic potential $\varphi(t, \boldsymbol{r})=\varphi(\boldsymbol{r})$ or a vector potential
$\boldsymbol{A}(t, \boldsymbol{r})=\boldsymbol{A}(t)=-\boldsymbol{A}(-t)$ which is an odd function of time only], the Euclidean potential $A_{\mu}$ and field tensor $F_{\mu \nu}$ are purely imaginary, so $i A_{\mu}$ and $i F_{\mu \nu}$ are real. In general they have both real and imaginary parts, though.

We immediately introduce dimensionless quantities using some reference field strength $E$, which makes a numerical treatment possible and simplifies the following derivation:

$\tilde{x}_{\mu}=x_{\mu} \frac{q E}{m}, \quad \tilde{F}_{\mu \nu}=F_{\mu \nu} \frac{1}{E}, \quad \tilde{A}_{\mu}=\frac{q E}{m} \frac{1}{E} A_{\mu}$,

and also rescale the integration variable

$$
\tilde{T}=q E T, \quad u=\frac{1}{T} \tau=\frac{q E}{\tilde{T}} \tau, \quad \curvearrowright \frac{\partial}{\partial u}=\frac{\tilde{T}}{q E} \frac{\partial}{\partial \tau} .
$$

We can now exchange the order of integration,

$$
\begin{aligned}
\Gamma= & \int_{x(1)=x(0)} \mathcal{D} x(u) \int_{0}^{\infty} \mathrm{d} \tilde{T} \frac{\Phi[\tilde{x}]}{\tilde{T}} \\
& \times \exp \left(-\frac{m^{2}}{q E}\left(\frac{\tilde{T}}{2}+\int_{0}^{1} \mathrm{~d} u\left(\frac{\dot{\tilde{x}}^{2}}{2 \tilde{T}}+i \dot{\tilde{x}} \cdot \tilde{A}\right)\right)\right),
\end{aligned}
$$

so we can perform the $\tilde{T}$-integration using Laplace's method, where, due to our rescaling, $\mathrm{m}^{2} / q E$ is singled out as the large parameter of the expansion, while all other quantities are assumed to be of order unity. We obtain the saddle point $\tilde{T}_{0}=\sqrt{\int_{0}^{1} \mathrm{~d} u \dot{\tilde{x}}^{2}}=: a[\tilde{x}]$, so including the quadratic fluctuation around the saddle we arrive at the approximation

$$
\Gamma \approx \int_{x(T)=x(0)} \mathcal{D} x(\tau) \sqrt{\frac{2 \pi}{a[\tilde{x}]} \frac{q E}{m^{2}}} \Phi[\tilde{x}] e^{-\frac{m^{2}}{q E} \mathcal{A}[\tilde{x}]},
$$

with the nonlocal (due to $a[\tilde{x}]$ ) action

$$
\mathcal{A}[\tilde{x}]=a[\tilde{x}]+\int_{0}^{1} \mathrm{~d} u \dot{\tilde{x}} \cdot i \tilde{A}(\tilde{x}),
$$

and the spin factor

$$
\Phi[\tilde{x}]=\frac{1}{2} \operatorname{tr} \mathcal{P} \exp \left(\frac{a[\tilde{x}]}{4} \int_{0}^{1} \mathrm{~d} u \sigma_{\mu \nu} i \tilde{F}_{\mu \nu}(\tilde{x})\right) .
$$

Note that in (10) we symbolically restored the original parametrization $\mathcal{D} x(\tau)$ in the path integral differential, this will be relevant for the discretization in the next section.

Applying Laplace's method (based on $m^{2} \gg q E$ ) to the path integral, we need to find a path $\tilde{x}_{\mu}(u)$ that satisfies the periodic boundary conditions and extremizes the exponent in (10), so a solution to the Euler-Lagrange equations (the Lorentz force equation in this case)

$$
\frac{\ddot{\tilde{x}}_{\mu}}{a[\tilde{x}]}=i q \tilde{F}_{\mu \nu} \dot{\tilde{x}}_{\nu}
$$


Contracting (13) with $\dot{\tilde{x}}_{\mu}$ we see that (due to the antisymmetry of $\tilde{F}_{\mu \nu}$ ) $\dot{\tilde{x}}^{2}=$ const $=a^{2}$, simplifying the instanton equations of motion to

$$
\ddot{\tilde{x}}_{\mu}=i a q \tilde{F}_{\mu \nu} \dot{\tilde{x}}_{\nu} .
$$

The prefactor of the Laplace approximation is given by the second variation of the action around the classical solution to (14), amounting to an operator determinant. The determinant has to be defined carefully, but we can completely sidestep this complication by instead performing Laplace's method after discretization, when we can calculate the fluctuation prefactor by standard methods of linear algebra.

\section{DISCRETIZATION}

We approximate (10) by discretizing the trajectories $\tilde{x}_{\mu}(u)$ into $N d$-dimensional points (in general $d=$ $3+1$, but for simple field configurations it is possible to only consider $d=1+1$ or $d=2+1$ dimensions, so we will keep the dimensionality variable):

$$
\tilde{x}_{\mu}^{i}:=\tilde{x}_{\mu}\left(\frac{l}{N}\right), \quad l=0,1, \ldots, N-1 .
$$

The velocity is then approximated using (forward) finite differences

$$
\dot{\tilde{x}}_{\mu}^{l} \approx \frac{\tilde{x}_{\mu}^{l+1}-\tilde{x}_{\mu}^{l}}{\varepsilon}, \quad \varepsilon=\frac{1}{N},
$$

with the identification $\tilde{x}_{\mu}^{N} \equiv \tilde{x}_{\mu}^{0}$.

Discretizing the path integral requires a normalization factor for each $\tilde{x}_{\mu}^{i}$ integral. We could find these factors by performing the integration in the free case, however this is not necessary: In the derivation of (4) the path integral arises as an ordinary nonrelativistic transition amplitude, so we can use Feynman's normalization 1/A for each integral (see, e.g., [32]), with

$$
A=\sqrt{\frac{2 \pi T_{0}}{N}}=\sqrt{\frac{2 \pi a[x]}{q E N}} .
$$

Using this normalization and replacing the $N \times d$ integrations by the dimensionless versions we arrive at the discretized worldline path integral

$$
\begin{aligned}
\Gamma \approx & \left(\prod_{k=0}^{N-1} \int \mathrm{d}^{d} \tilde{x}^{k}\right)\left(\frac{m^{2}}{q E} \frac{N}{2 \pi a[\tilde{\boldsymbol{X}}]}\right)^{N d / 2} \\
& \times \sqrt{\frac{2 \pi}{a[\tilde{\boldsymbol{X}}]} \frac{q E}{m^{2}} \Phi[\tilde{\boldsymbol{X}}] e^{-\frac{m^{2}}{q E} \mathcal{A}[\tilde{\boldsymbol{X}}]} .}
\end{aligned}
$$

To ease notation, we have condensed the proper time index $l$ and the spacetime index $\mu$ into a single vector

$$
\tilde{\boldsymbol{X}}=\left(\tilde{x}_{1}^{0}, \tilde{x}_{2}^{0}, \ldots, \tilde{x}_{d}^{0}, \tilde{x}_{1}^{1}, \ldots, \tilde{x}_{d}^{N-1}\right),
$$

where the square brackets $[\tilde{\boldsymbol{X}}]$ denote dependence on all points $\tilde{x}_{\mu}^{l}$ with $\mu=1, \ldots, d$ and $l=1, \ldots, N$.

As we have now expressed everything in terms of the dimensionless variables, we will from now on drop the tilde. We still need discretized expressions for $a, \mathcal{A}$ and $\Phi$,

$$
a[\boldsymbol{X}]:=\sqrt{N \sum_{k=0}^{N-1}\left(x_{\nu}^{k+1}-x_{\nu}^{k}\right)^{2}}
$$

$$
\begin{aligned}
\mathcal{A}[\boldsymbol{X}]:= & a[\boldsymbol{X}] \\
& +\sum_{k=0}^{N-1}\left(\frac{A_{\nu}\left(x_{\mu}^{k+1}\right)+A_{\nu}\left(x_{\mu}^{k}\right)}{2}\right)\left(x_{\nu}^{k+1}-x_{\nu}^{k}\right) .
\end{aligned}
$$

The form of discretization of the gauge term is not at all obvious, other choices like having just $A_{\nu}\left(x_{\mu}^{k}\right)$ or $A_{\nu}\left(x_{\mu}^{k+1}\right)$ or evaluating the gauge field between points $A_{\nu}\left(\left(x_{\mu}^{k}+\right.\right.$ $\left.x_{\mu}^{k+1}\right) / 2$ ) would yield the same classical continuum limit. That does not mean however that the resulting propagator is the same, see [33-36]. The midpoint prescription in (21) arises when the path integral representation is derived from the vacuum persistence amplitude using the time slicing procedure, see e.g., [37].

Special care has to be taken to define a discretized expression for the spin factor that obeys path ordering. Instead of approximating the integral by summation and taking the exponential, we employ the product representation of the exponential function which is automatically path ordered (cf. [38]),

$$
\Phi[\boldsymbol{X}]:=\operatorname{tr}\left[\prod_{k=0}^{N-1}\left(1+\frac{a[\boldsymbol{X}]}{4 N} \sigma_{\mu \nu} i F_{\mu \nu}\left(x_{\lambda}^{k}\right)\right)\right] .
$$

The finite dimensional integral (18) can now be approximated using Laplace's method as well, by finding an $N \times d$-dimensional vector $\bar{x}_{\mu}^{l}$ (a discrete worldline instanton) that extremizes the action function $\mathcal{A}[\boldsymbol{X}]$, i.e.,

$$
\left.\frac{\mathrm{d} \mathcal{A}}{\mathrm{d} x_{\mu}^{l}}\right|_{x_{\mu}^{l}=\bar{x}_{\mu}^{l}}=0,
$$

so a discrete instanton $\bar{X}$ has the property

$$
\boldsymbol{F}[\overline{\boldsymbol{X}}]:=\left.\nabla \mathcal{A}[\boldsymbol{X}]\right|_{\overline{\boldsymbol{X}}}=\mathbf{0} .
$$

Equation (24) describes a system of $N \times d$ nonlinear equations in $N \times d$ unknowns, which can be solved numerically using the Newton-Raphson method or a similar root finding scheme. 
In this discretized picture, the fluctuation prefactor is readily computed as well, via the determinant of the Hessian of $\mathcal{A}$ :

$$
\mathbf{H}[\overline{\boldsymbol{X}}]=\left.(\nabla \otimes \nabla) \mathcal{A}[\boldsymbol{X}]\right|_{\overline{\boldsymbol{X}}},
$$

giving the full semiclassical approximation of the discretized worldline path integral

$$
\Gamma \approx \sqrt{\frac{2 \pi}{a^{\mathrm{cl}}} \frac{q E}{m^{2}}}\left(\frac{N}{a^{\mathrm{cl}}}\right)^{N d / 2} \frac{\Phi[\overline{\boldsymbol{X}}] e^{-\frac{m^{2}}{q E} \mathcal{A}[\overline{\boldsymbol{X}}]}}{\sqrt{\operatorname{det} \mathbf{H}[\overline{\boldsymbol{X}}]}},
$$

with $a^{\mathrm{cl}}:=a[\overline{\boldsymbol{X}}]$. If the function $\mathcal{A}[\overline{\boldsymbol{X}}]$ were entirely well behaved we would be done now, we would just need to find solutions of (24) and plug them into (26). The Gaussian integration resulting in the determinant prefactor however is only defined for positive definite matrices in the exponent, which our Hessian $\mathbf{H}$ is not.

\section{REGULARIZATION OF THE PREFACTOR}

We have two problems with the Hessian matrix of the action $\mathcal{A}$. One is that of negative eigenvalues of $\mathbf{H}$. The corresponding direction in the Gaussian integration diverges, and the integral has to be defined by analytic continuation. A single negative mode (which is present for a static electric field) thus turns the determinant negative, and the whole expression (26) imaginary. This could seem troubling at first, as the pair production is given by the real part of the Euclidean effective action. For a field not depending on time we expect a volume factor from the $x_{4}$ integration though, which has to be purely imaginary for a real temporal volume factor $V_{t}=-i V_{x_{4}}$.

A more serious technical issue is that of zero modes. One or more zero eigenvalues of $\mathbf{H}$ immediately spoil our result, so they have to be removed from the integration in some way. One zero mode that is always present in the worldline path integral is the one corresponding to reparametrization. Due to the periodic boundary conditions we can move every point of the curve along the trajectory without a change in action. We would thus like to separate the integration in this direction (resulting in a "volume factor" of the periodicity, in our rescaled expression just unity) from the other integrations.

We will use the Faddeev-Popov method [39] to perform this separation. While it is commonly used to remove gauge-equivalent configurations from a gauge theory path integral, it can be applied to this simpler scenario as well. We insert a factor of unity into the path integral in terms of the identity

$$
1=\frac{1}{w} \int \mathrm{d} \lambda \delta(\chi(\lambda))\left|\frac{\mathrm{d}}{\mathrm{d} \lambda} \chi(\lambda)\right|
$$

where $\chi(\lambda)$ is some function chosen so that $\chi=0$ fixes the zero mode, $\lambda$ parametrizes the symmetry and $w$ is the number of times $\chi(\lambda)=0$ occurs over the integration interval [40]. The idea is now that the $\lambda$ integration can be performed due to the symmetry of the path integral, resulting in the desired volume factor and a Dirac delta that fixes the corresponding mode. This is especially elegant for a discrete numerical evaluation of the semiclassical approximation, as we can use an exponential representation of the delta function

$$
\delta(\chi)=\lim _{\varepsilon \rightarrow 0} \sqrt{\frac{m^{2} / q E}{\varepsilon}} \exp \left(-\frac{\pi}{\varepsilon} \frac{m^{2}}{q E} \chi^{2}\right),
$$

where the Gaussian integration over the zero mode produces a factor of $\sqrt{\varepsilon}$ canceling the prefactor, enabling us to simply set $\varepsilon=1$. We insert the factor of $\mathrm{m}^{2} / q E$ for convenience, so the action $\mathcal{A}$ in (18) just gets an additional term $\pi \chi^{2}$.

To fix the reparametrization mode, we take (cf. [40-42])

$$
\chi_{u}\left(\lambda_{u}\right)=\frac{2}{\left(a^{\mathrm{cl}}\right)^{2}} \int_{0}^{1} \mathrm{~d} u \dot{x}_{\nu}^{\mathrm{cl}}(u) x_{\nu}\left(u+\lambda_{u}\right)
$$

which is chosen so that

$$
\frac{1}{w}\left|\chi_{u}^{\prime}(0)\right|=\frac{1}{2} \frac{2}{\left(a^{\mathrm{cl}}\right)^{2}} \int_{0}^{1} \mathrm{~d} u \dot{x}_{\nu}^{\mathrm{cl}}(u) \dot{x}_{\nu}(u) \stackrel{x=x^{\mathrm{cl}}}{=} 1,
$$

at the saddle point. Due to the translation invariance we can set $\lambda_{u}=0$ in the integrand so the $\lambda_{u}$ integration is equal to one. This means we only need to add the (discretized version of) $\chi_{u}(0)$ to the action as in (28), the second derivatives to $\mathbf{H}$ and a factor of $\sqrt{m^{2} / q E}$ from (28) to the prefactor, and just proceed as if no zero mode were present.

Other zero eigenvalues appear if the electric background field does not depend on all spacetime coordinates. They are of course easier to deal with, we could just omit the corresponding integrals and add a volume factor $\tilde{L}_{\mu}$ (the tilde is to stress that this is in terms of the dimensionless coordinates) per invariant direction $x_{\mu}$. We can, however, treat these just as the reparametrization direction, which simplifies a numerical implementation that supports arbitrary fields. Choosing $\chi_{\mu}$ to be the average of $x_{\mu}$ along the trajectory we obtain the volume $\tilde{L}_{\mu}$, and again a factor of $\sqrt{m^{2} / q E}$.

To summarize, our final expression for the semiclassical approximation of the effective action is

$$
\Gamma \approx \frac{V_{N_{0}}}{m^{-N_{0}}}\left(\frac{q E}{m^{2}}\right)^{\frac{N_{0}}{2}} \sqrt{\frac{2 \pi}{a^{\mathrm{cl}}}}\left(\frac{N}{a^{\mathrm{cl}}}\right)^{\frac{N d}{2}} \frac{\Phi[\overline{\boldsymbol{X}}] e^{-\frac{m^{2}}{q E} \mathcal{A}[\overline{\boldsymbol{X}}]}}{\sqrt{\operatorname{det} \mathbf{H}[\overline{\boldsymbol{X}}]}},
$$

where the appropriate terms of $\chi$ and its derivatives have been added to $\mathcal{A}$ and $\mathbf{H}, N_{0}$ is the number of invariant 
spacetime directions, and $V_{N_{0}}$ the corresponding volume factor (with units reinstated). Note that (31) unambiguously contains the full prefactor including spin effects for an arbitrary background field, without having to resort to limiting cases to determine any normalization constants. This demonstrates an advantage of our discretized approach: In a continuum description, special care is required in order to collect all the minus signs correctly (e.g., by calculating the Morse index in order to obtain the correct phase, as in [23]). In our approach, the Hessian $\mathbf{H}$ is discrete and thus has a well-defined number of positive, vanishing, and negative eigenvalues - which automatically yield the correct phase [43].

In addition, the reference field strength $E$ enters only in the combination $q E / \mathrm{m}^{2}$ in front of the action and in the prefactor, which has two advantages. First, having found an instanton $\overline{\boldsymbol{X}}$, we can evaluate (31) for arbitrary values of $q E / m^{2}$ without any additional computational effort. Second, the accuracy of the discretization does not depend on the field strength, so there are no numerical instabilities for small $E$.

Figure 1 shows how the discretization error scales with the number of points $N$ for a constant, homogeneous electric field. For scalar QED (that is, without the spin factor $\Phi$ ) the error in the prefactor decreases as $N^{-1}$ as expected for a first order discretization procedure. As the first variation of the action vanishes for an instanton, the error of the exponent even decreases as $N^{-2}$. For spinor QED, on the other hand, the error in the prefactor decreases as $N^{-2}$ as well. The reason for this is not obvious, as the only difference is an additional, seemingly independent multiplicative spin factor.

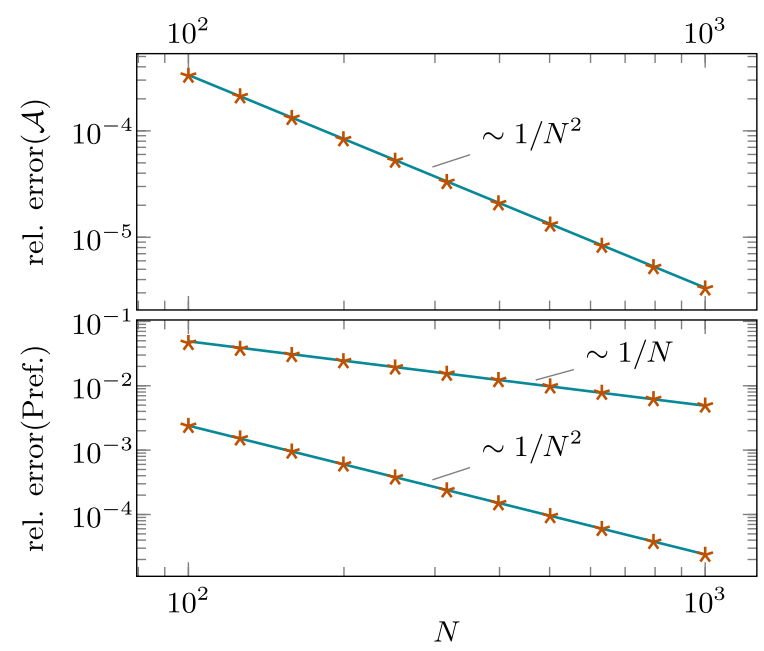

FIG. 1. Accuracy of the method for a constant, homogeneous field. The error of the prefactor decreases as $1 / N$ (the first order discretization error) for scalar QED (upper markers in the bottom plot), the error of the action as $1 / N^{2}$ (because the action has an extremum at that point, upper plot). Interestingly, for spinor QED the prefactor decreases as $1 / N^{2}$ as well (lower markers).

\section{NUMERICAL CONTINUATION}

For most fields we are interested in, there is one (or multiple) parameter that we would like to vary, for example the timescale of a pulsed field or the inhomogeneity of a spatially varying field configuration. Let us denote such a parameter $\gamma$. In general we are interested in the full family of instantons $\overline{\boldsymbol{X}}(\gamma)$. Methods to numerically map such a solution space are known as numerical continuation algorithms [44,45].

If we know an instanton for a particular value $\gamma_{i}$ of the parameter (e.g. the limit $\omega \rightarrow 0$ for a time-dependent pulse), we can use it as the starting point for the numerical solution of (24) for a parameter value $\gamma_{i+1}=\gamma_{i}+\Delta \gamma$, which is the method used in [46]. If we choose a sufficiently small $\Delta \gamma$, we can expect the root finding procedure to quickly converge. This process is called natural parameter continuation, because we vary a physical parameter of the problem at hand, instead of introducing an artificial variable to blend between an easy and our actual problem [e.g. solving $\left.0=\boldsymbol{G}(\boldsymbol{X}, \gamma):=\gamma \boldsymbol{F}(\boldsymbol{X})+(1-\gamma) \boldsymbol{F}_{0}(\boldsymbol{X})\right]$.

Natural parameter continuation works well if the solutions $\overline{\boldsymbol{X}}(\gamma)$ depend on the parameter in a smooth and uniform manner. If, however, the dependence on $\gamma$ varies strongly, it is difficult to choose appropriate step lengths $\Delta \gamma$. For some spatially inhomogeneous fields the instantons even grow infinitely large in some limit $\gamma \rightarrow \gamma^{\text {crit }}$, so we need to take ever smaller steps to reach this value. We could, in principle, adaptively adjust the step length when the root finding for the next parameter value converges poorly, but there is an easier method of choosing the increment $\Delta \gamma$.

Natural parameter continuation can be viewed as a predictor-corrector scheme, with the "zeroth-order" predictor step of just taking the last solution as the starting point for the next parameter, and performing the numerical root finding as a corrector step. We can find a better prediction by taking the $\gamma$ derivative of (24), yielding the Davidenko differential equation [47]:

$$
\mathbf{0}=\frac{\mathrm{d}}{\mathrm{d} \gamma} \boldsymbol{F}(\overline{\boldsymbol{X}}, \gamma)=\mathbf{H}(\overline{\boldsymbol{X}}, \gamma) \cdot \frac{\mathrm{d} \overline{\boldsymbol{X}}}{\mathrm{d} \gamma}+\frac{\partial}{\partial \gamma} \boldsymbol{F}(\overline{\boldsymbol{X}}, \gamma)
$$

and thus, provided that $\mathbf{H}$ is invertible (which it is by our regularization scheme),

$$
\frac{\mathrm{d} \overline{\boldsymbol{X}}}{\mathrm{d} \gamma}=-(\mathbf{H}(\overline{\boldsymbol{X}}, \gamma))^{-1} \cdot\left(\frac{\partial}{\partial \gamma} \boldsymbol{F}(\overline{\boldsymbol{X}}, \gamma)\right) .
$$

We can now use (33) in two ways: first, having found an instanton $\overline{\boldsymbol{X}}_{i}$ for a parameter value $\gamma_{i}$, it tells us in which way the instanton for a slightly different value of $\gamma$ differs from the current one, so we can use it as a much improved predictor in our predictor-corrector scheme, i.e., $\overline{\boldsymbol{X}}_{i+1} \approx$ $\overline{\boldsymbol{X}}_{i}+\Delta \gamma \mathrm{d} \overline{\boldsymbol{X}} / \mathrm{d} \gamma$. In fact, we could directly integrate (33) to 
obtain all solutions. Unfortunately, evaluating the Hessian is costly and we can afford a much larger step size by performing the corrector steps. As a compromise it is possible to perform multiple steps according to (33) before starting the root finding routine. Furthermore, we can use the derivative to scale the step $\Delta \gamma$ by instead specifying a maximum (or mean) difference between the points of $\overline{\boldsymbol{X}}_{i}$ and the proposed guess for $\overline{\boldsymbol{X}}_{i+1}$, or even a fixed arclength $\Delta s$ of the solution curve in $\mathbb{R}^{N \times d+1}$,

$$
\begin{aligned}
\Delta s & =\sqrt{(\Delta \gamma \mathrm{d} \overline{\boldsymbol{X}} / \mathrm{d} \gamma)^{2}+(\Delta \gamma)^{2}} \\
\Leftrightarrow \Delta \gamma & =\frac{\Delta s}{\sqrt{(\mathrm{d} \overline{\boldsymbol{X}} / \mathrm{d} \gamma)^{2}+1}} .
\end{aligned}
$$

A situation may be conceivable where it is not possible to parametrize the solution set as $\overline{\boldsymbol{X}}(\gamma)$ at all, because such a function would not be single valued or have infinite slope somewhere. In this case, we can parametrize both the solution and the parameter $\gamma$ by a new parameter

$$
\begin{aligned}
& \overline{\boldsymbol{Y}}(u)=(\overline{\boldsymbol{X}}(u), \gamma(u))^{\top} \\
& \Rightarrow 0=\frac{\mathrm{d}}{\mathrm{d} u} \tilde{\boldsymbol{F}}(\overline{\boldsymbol{Y}})=\tilde{\mathbf{H}} \cdot \frac{\mathrm{d} \overline{\boldsymbol{Y}}}{\mathrm{d} u},
\end{aligned}
$$

where $\tilde{\mathbf{H}}$ is now an $(N d+1) \times(N d)$ matrix, so (35) has to be augmented by an additional condition. This is chosen to be a constraint on the orientation and the "velocity" of the flow $1=\|\mathrm{d} \overline{\boldsymbol{Y}} / \mathrm{d} u\|$, so $\overline{\boldsymbol{Y}}(u)$ is parametrized by arclength, hence the name pseudo-arclength continuation (pseudo because this is only approximately true, as we are taking discrete steps). As long as $\gamma$ is a suitable parameter, this is equivalent to (34), which is what we will be using in the following.

\section{APPLICATIONS}

Let us now apply the method outlined above to some background fields. The strategy in all cases is to start with a limit that is reasonably close to a static, homogeneous field and perform pseudo-arclength continuation to map the solution space for a chosen parameter range. In all figures depicting worldline instantons we color the homogeneous limit (i.e., a circular instanton) purple, and all further instantons proportional to the change in action (blue for a decrease, red for an increase, so blue means more, red less pair production). In all figures that show the full effective action we choose $E=0.033 \mathrm{~m}^{2} / q$ for the reference field strength. This is simply the value we already used in earlier works, and it does not influence the quality of the discretization in any way. We also use $N=500$ points in the discretization, which yields good accuracy while it still takes less than thirty seconds to obtain the family of instantons in the cases below, with the exception of the $e$-dipole pulse.

\section{A. Temporal Sauter pulse}

First let us consider simple, one-dimensional inhomogeneities where we can compare to analytic results. As an example, we choose the Euclidean four-potential $i A_{3}=\tan \left(\gamma_{\omega} x_{4}\right) / \gamma_{\omega}$ describing the (physical) field $\boldsymbol{E}=$ $E \cosh ^{-2}(\omega t) \boldsymbol{e}_{z}$ with the Keldysh parameter $\gamma_{\omega}=m \omega / q E$ [48]. Since the field does not depend on any spatial coordinates, we have $N_{0}=3$ translational zero modes that need to be held fixed.

The analytical worldline instanton result for this field is [23]

$$
\begin{aligned}
\frac{\Im \Gamma_{\mathrm{M}}^{\mathrm{Sauter}^{\omega}}}{V_{3}}= & \frac{(q E)^{3 / 2}}{(2 \pi)^{3}} \frac{\left(1+\gamma_{\omega}^{2}\right)^{5 / 4}}{\gamma_{\omega}} \\
& \times \exp \left(-\frac{m^{2} \pi}{q E} \frac{2}{1+\sqrt{1+\gamma_{\omega}^{2}}}\right) .
\end{aligned}
$$

In Fig. 2 the first plot shows a family of instantons in the range of $0<\gamma_{\omega}<3.5$, and the top panel in Fig. 3 compares the numerical result (31) in this parameter range to the analytical expression (36), showing near-perfect agreement.

\section{B. Spatial Sauter pulse}

We can also consider the spatially inhomogeneous profile $i A_{4}=\tanh \left(\gamma_{k} x_{3}\right) / \gamma_{k}$ describing the (physical) field $\boldsymbol{E}=E \cosh ^{-2}(k z) \boldsymbol{e}_{z}$ with (the spatial analog of) the Keldysh parameter $\gamma_{k}=m k / q E$. The analytical result is related to (36) by $\gamma_{\omega} \rightarrow i \gamma_{k}$ [23],

$$
\begin{aligned}
\frac{\Im \Gamma_{\mathrm{M}}^{\text {Sauter }}{ }^{k}}{V_{t} V_{2}}= & \frac{(q E)^{3 / 2}}{(2 \pi)^{3}} \frac{\left(1-\gamma_{k}^{2}\right)^{5 / 4}}{\gamma_{k}} \\
& \times \exp \left(-\frac{m^{2} \pi}{q E} \frac{2}{1+\sqrt{1-\gamma_{k}^{2}}}\right),
\end{aligned}
$$

where the instanton is now confined in the $x_{3}$ direction and we obtain a "temporal volume factor" $V_{t}$ instead. The worldline instantons in this field for the range $0<\gamma_{k}<1$ are depicted in the middle of Fig. 2, and the comparison of the numeric result and the analytic expression (37) in the bottom panel of Fig. 3 .

\section{Spacetime Sauter pulse}

As a simple example of a both space- and time-dependent background we choose the product of the preceding profiles with $\gamma:=\gamma_{\omega}=\gamma_{k} / 3$, i.e., $i A_{3}=\cosh ^{-2}\left(3 \gamma x_{3}\right) \tan \left(\gamma x_{4}\right) / \gamma$. The resulting worldline instantons in the range $0<\gamma<2.5$ are shown in the bottom plot of Fig. 2 and in Fig. 4, and the resulting pair production rate in Fig. 5. The minimum in Fig. 5 at $\gamma \approx 1 / 2$ reflects the competition between the impact of the temporal dependence, which tends to increase the pair-creation probability, cf. Eq. (36), and the spatial 

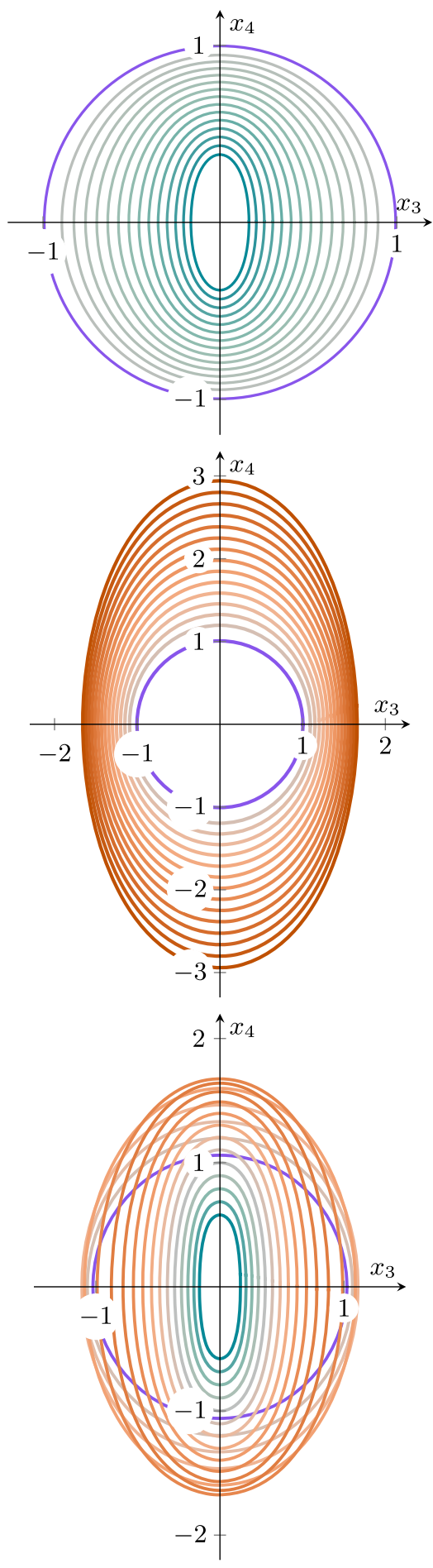

FIG. 2. Planar instantons for multiple background fields and increasing values of $\gamma_{\omega / k}$. Top: temporal Sauter field $\boldsymbol{E}=$ $E \cosh ^{-2}(\omega t) \boldsymbol{e}_{z}$; middle: spatial Sauter field $\boldsymbol{E}=E \cosh ^{-2}(k z) \boldsymbol{e}_{z}$; bottom: spacetime bump profile $\boldsymbol{E}=E \cosh ^{-2}(\omega t) \cosh ^{-2}(k z) \boldsymbol{e}_{z}$ with $k=3 \omega$. The purple trajectories are the limit $\gamma_{\omega / k} \rightarrow 0$, blue denotes a decrease in action, red an increase. As is well known, while temporal variation shrinks the instantons and decreases the worldline action (top), spatial inhomogeneity has the opposite effect (middle). As the bottom plot shows, field configurations are possible that both increase and decrease the action in different regimes.

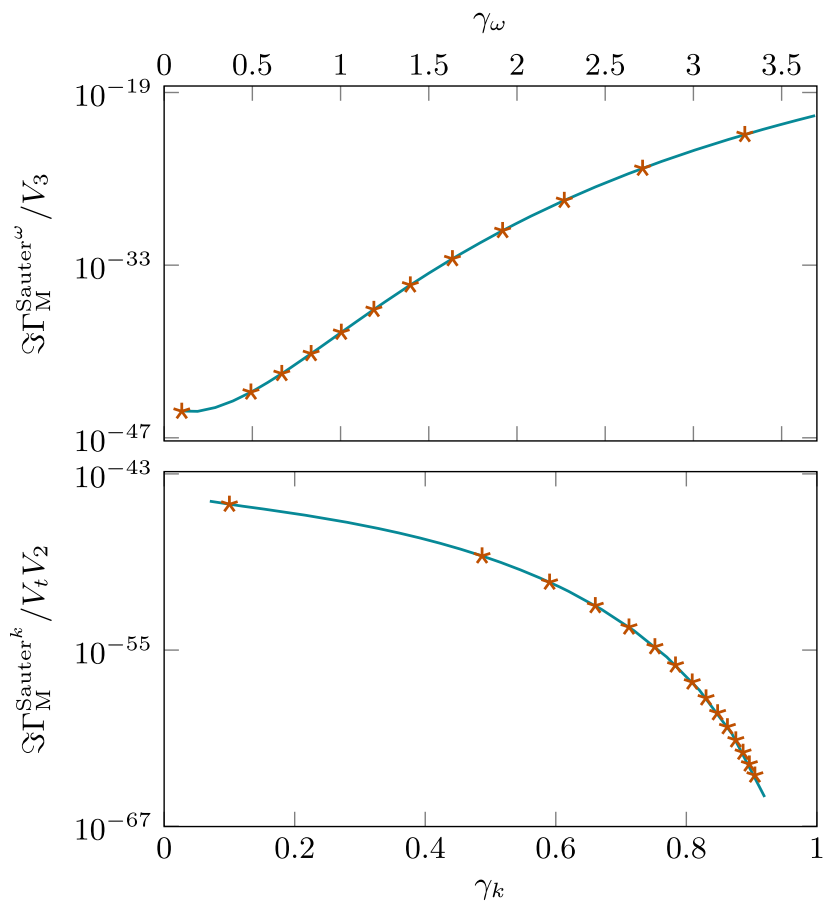

FIG. 3. Imaginary part of the Minkowskian effective action (i.e., the pair production rate) for $E=0.033 \mathrm{~m}^{2} / q$. Top: temporal Sauter pulse; bottom: spatial Sauter profile. Numerical results are given by markers and the analytic expressions (36) and (37) by lines. Note the spacing of markers in the spatial case, the step length decreases to keep the overall arclength $\Delta s$ constant.

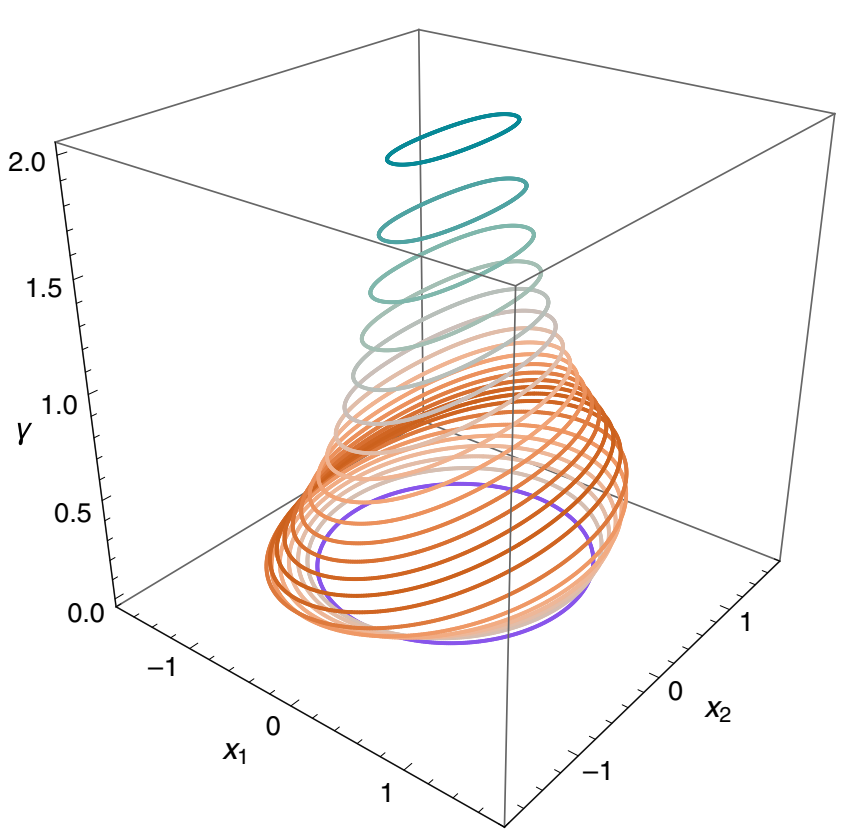

FIG. 4. The same worldline instantons as in the third plot of Fig. 2, but stacked, with the $z$-coordinate given by the parameter $\gamma$. This presentation makes it easier to correlate the instantons' change in shape with the parameter. 


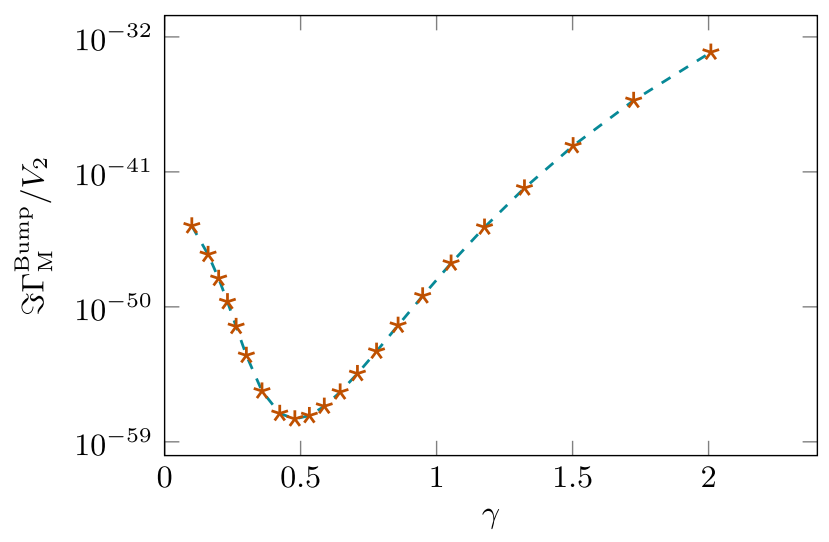

FIG. 5. Imaginary part of the Minkowskian effective action for the spacetime bump profile $\boldsymbol{E}=E \cosh ^{-2}(\omega t) \cosh ^{-2}(k z) \boldsymbol{e}_{z}$ with $k=3 \omega$ and $E=0.033 m^{2} / q$. Here (and in the following cases) there are no analytical results to compare with, so we just add the connecting dashed lines as a guide to the eye.

dependence, which tends to decrease it, cf. Eq. (37). With the chosen ratio $\gamma_{k} / \gamma_{\omega}=3$, these two opposite effects operate on different scales. For small $\gamma$, the spatial inhomogeneity dominates and results in shrinking instantons with a decreasing action and a growing effective volume, leading to an increasing pair-creation probability, as $\gamma$ goes to zero. For large $\gamma$, on the other hand, the temporal dependence dominates and produces smaller instantons, reduced action and increased pair production as $\gamma$ grows.

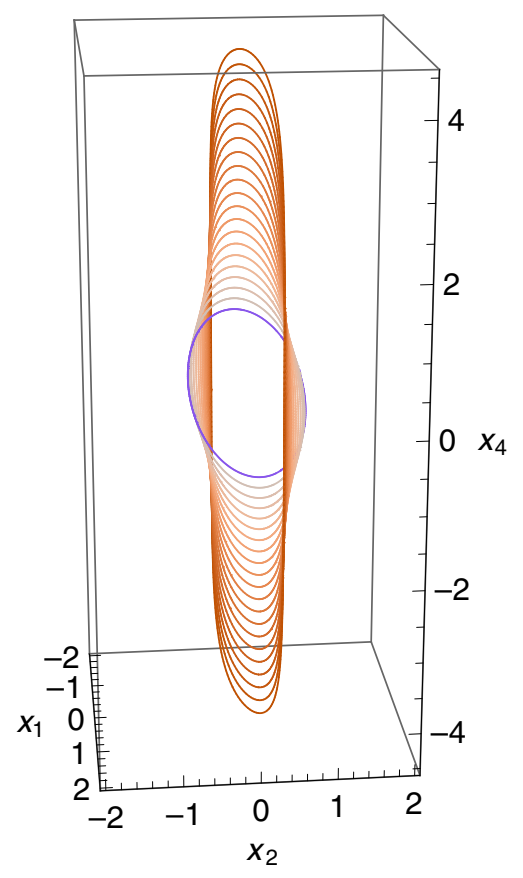

FIG. 6. Worldline instantons for the four-potential (38) from [28]. As before, stronger inhomogeneity stretches the instantons (in a more complicated way than for the one-dimensional fields) and increases the action.
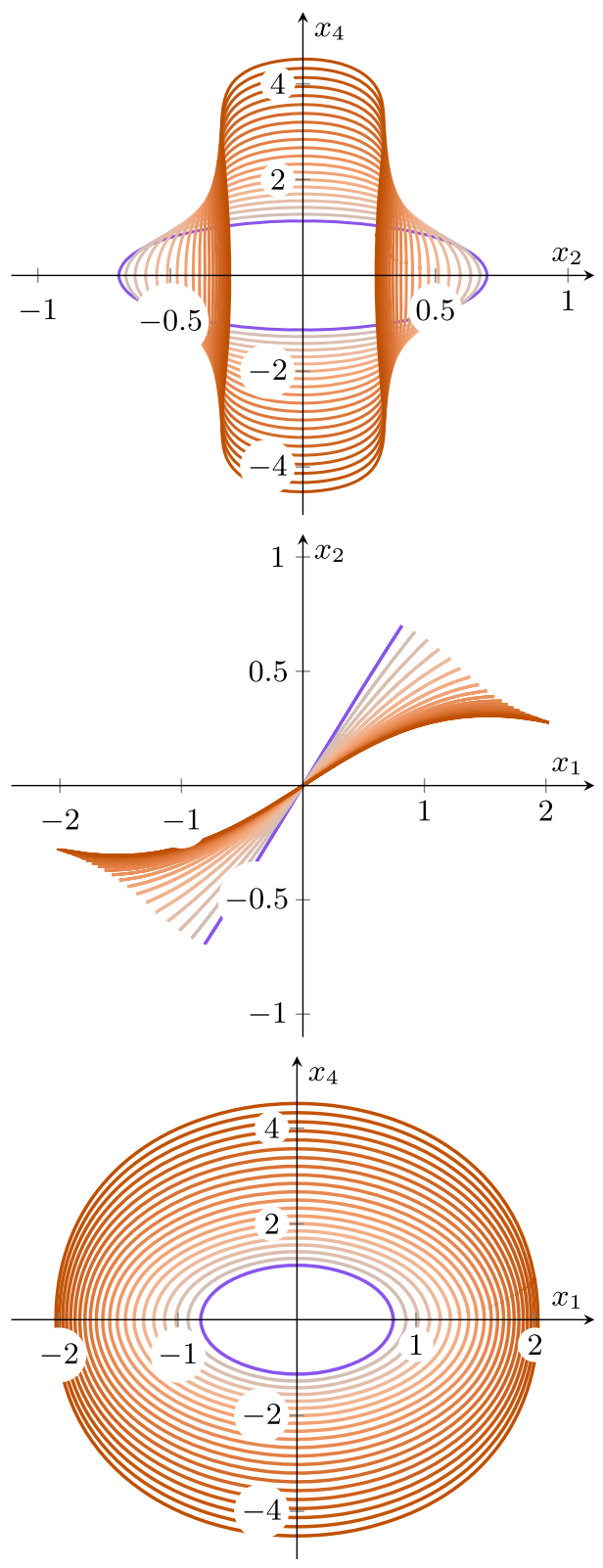

FIG. 7. The same worldline instantons as in Fig. 6, projected onto the coordinate planes.

\section{Multidimensional instantons}

In [28] multidimensional instantons were found for background fields that depend on multiple spatial coordinates using the shooting method. We can obtain instantons for these fields using discretization as well. Consider the potential

$$
i A_{4}=\frac{1}{\sqrt{2} k} \frac{\tanh \left(k x_{1}+k x_{2}\right)}{1+\left(k x_{1}\right)^{2}+10\left(k x_{2}\right)^{2}}
$$

from Fig. 1 in [28] (with the factor of $1 / \sqrt{2}$ added so the peak strength is 1 ). This yields three-dimensional instantons 


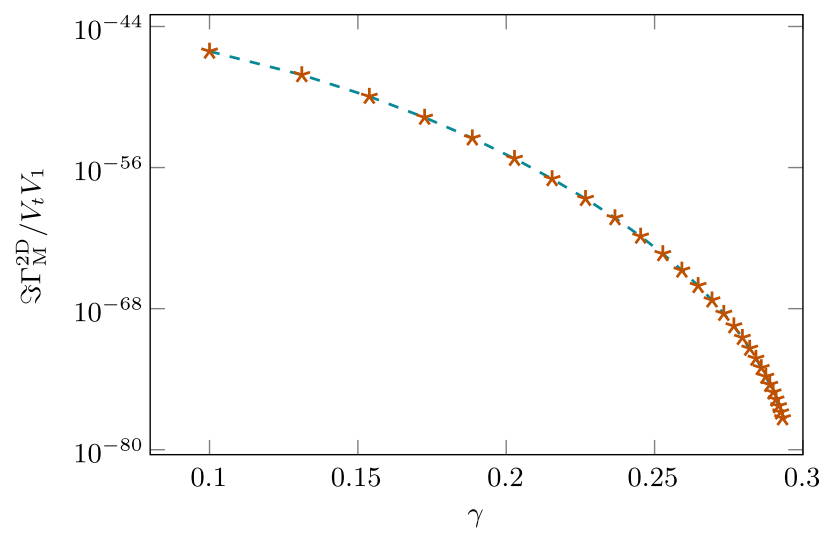

FIG. 8. Imaginary part of the effective action for the multidimensional field from [28] for $E=0.033 \mathrm{~m}^{2} / q$. The dashed, connecting line is again a visual aid only.

(in the $x_{1}, x_{2}$ and $x_{4}$ direction). Figure 6 depicts a family of instantons in a three-dimensional plot, while Fig. 7 shows all two-dimensional projections of the same trajectories. The resulting pair production rate is given in Fig. 8.

\section{E. Plane wave plus electric field}

In [49] we already applied the discrete worldline instanton method to calculate the pair creation rate for the superposition of a weak propagating plane wave and a constant field, a variant of dynamically assisted pair

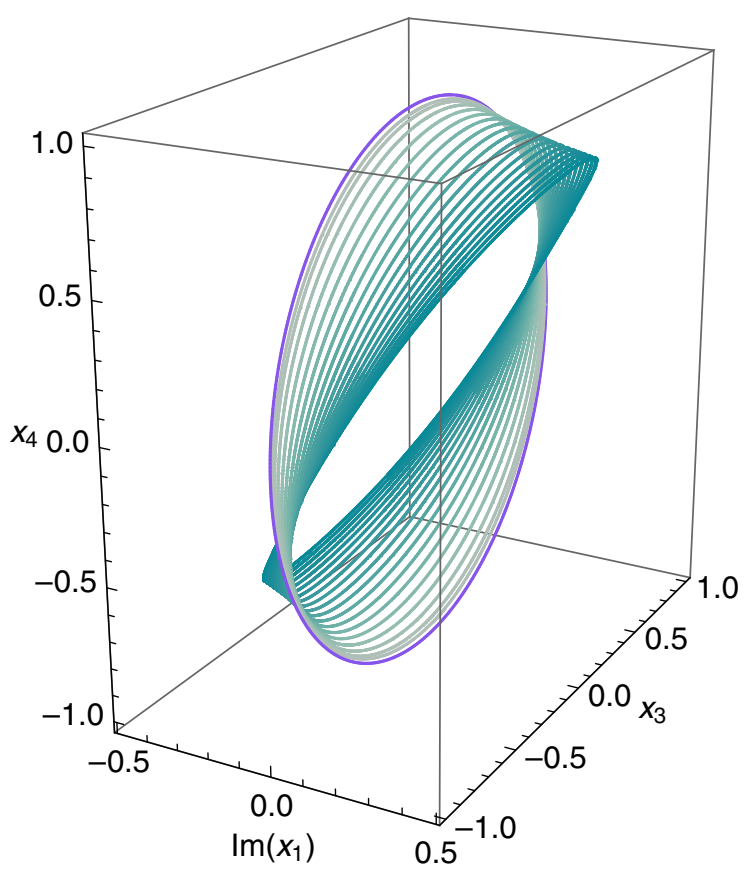

FIG. 9. Worldline instantons for the superposition of a static, homogeneous field and a weak, propagating plane wave. The ratio of the plane wave amplitude and the strong field is $10^{-2}$. The $x_{1}$ component of the trajectories is purely imaginary, hence the imaginary part of $x_{1}$ on the first axis.
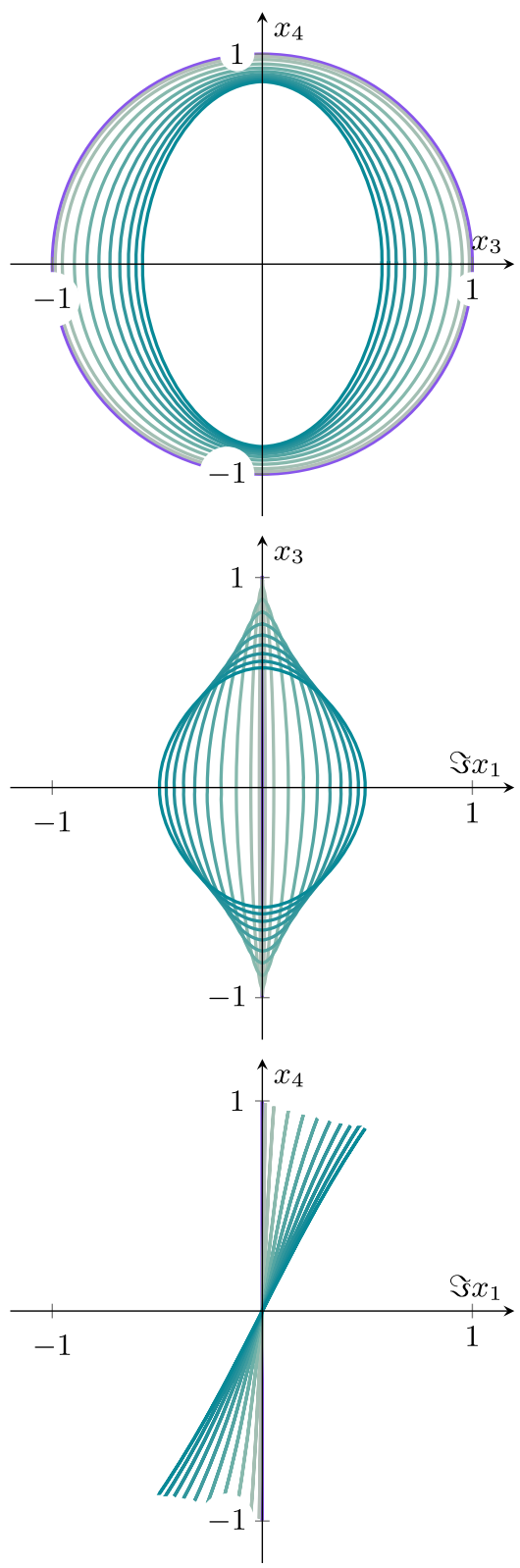

FIG. 10. The same worldline instantons as in Fig. 9, projected onto the coordinate planes.

production [25]. Different pulse shapes have been considered for the weak field before [50], however a plane wave is special in that it cannot produce pairs on its own, so the process is fully nonperturbative for all frequencies. In the case of parallel polarization (the plane wave and the constant field point in the same direction, but perpendicular to the propagation direction) this combination can be represented by the four-potential

$$
i A_{4}=x_{3}, \quad i A_{3}=i \frac{\varepsilon}{\gamma} \sin \left(\gamma\left(x_{1}-i x_{4}\right)\right) .
$$

The method can handle the perpendicularly polarized case just as well, however that leads to four-dimensional instantons that are cumbersome to visualize. 


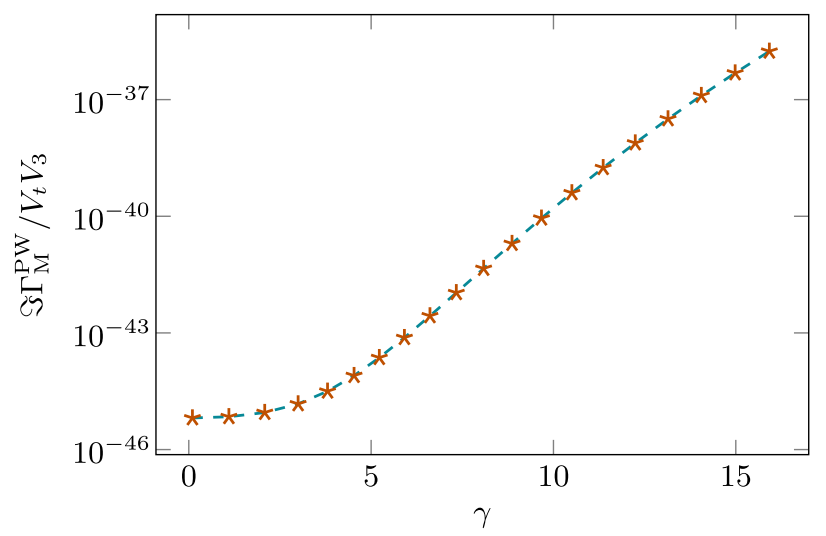

FIG. 11. Pair production rate for a constant field $\left(E_{\text {Strong }}=\right.$ $\left.0.033 \mathrm{~m}^{2} / q\right)$ with superimposed plane wave $\left(E_{\text {Weak }}=\right.$ $\left.10^{-2} E_{\text {Strong }}\right)$. The temporal volume factor $V_{t}$ arises from the number of instantons, one per oscillation of the wave at a fixed spatial point. The dashed line is added as a guide to the eye.

In contrast to the examples considered before, the field (39) leads to complex instantons, in particular purely real $x_{3}(u), x_{4}(u)$ and purely imaginary $x_{1}(u)$. A family of instantons is shown in Figs. 9 and 10, while the full pair production rate is given in Fig. 11.

\section{F. E-dipole pulse}

An especially interesting, highly nontrivial example is that of an e-dipole pulse. It is a solution to Maxwell's equations in vacuum that represents a localized pulse of finite energy [51]. It saturates the theoretical upper bound of peak field strength for given laser power [52] and is thus in a sense the optimal (and at the same time physically viable) configuration to study pair creation [53]. Its name stems from the structural similarity to dipole radiation, however it does not suffer from the strong singularities at the origin for a simple nonstationary dipole.

The electromagnetic field of the $e$-dipole pulse can be given in terms of a driving function $g$ using the vector $\boldsymbol{Z}$ [53]:

$$
\begin{aligned}
& \boldsymbol{Z}=\boldsymbol{e}_{z} \frac{d}{|\boldsymbol{r}|}(g(t+|\boldsymbol{r}|)-g(t-|\boldsymbol{r}|)), \\
& \boldsymbol{E}=-\nabla \times(\nabla \times \boldsymbol{Z}), \quad \boldsymbol{B}=-\nabla \times \dot{\boldsymbol{Z}} .
\end{aligned}
$$

We choose the function

$$
g(t)=\frac{t}{4 \omega^{2}} e^{-\omega^{2} t^{2}}+\frac{\sqrt{\pi}}{8 \omega^{3}}\left(1+2 \omega^{2} t^{2}\right) \operatorname{erf}(\omega t)
$$

and the virtual dipole moment $d=3 E / 4$, so that at the origin

$$
\boldsymbol{E} \approx E e^{-\omega^{2} t^{2}} \boldsymbol{e}_{z}
$$

We cannot immediately apply the instanton approach to this field since it is not given in terms of a four-potential. It is however possible to obtain an expression for the potential in coordinate gauge $A(x) \cdot x=0$ from the field tensor [54],

$$
A_{\mu}^{\mathrm{M}}(x)=-\int_{0}^{1} \mathrm{~d} \alpha F_{\mu \nu}^{\mathrm{M}}(\alpha x) \alpha x^{\nu} .
$$

For the field (40) this gives a lengthy expression, which can now be used to obtain worldline instantons.

Figure 12 shows the result. The top plot compares the instanton action for the $e$-dipole pulse (markers) to the action for a spatially constant field with only Gaussian time dependence, i.e., (42) (line). Due to the additional spatial inhomogeneity in the $e$-dipole field the action is slightly larger (and thus pair production slightly lower) than for the purely time dependent pulse. We can also compare the full imaginary part of the effective action with the locally constant field approximation, where the constant field result (1) with $n=1$ is assumed to hold at each spacetime point (see e.g. [53]),

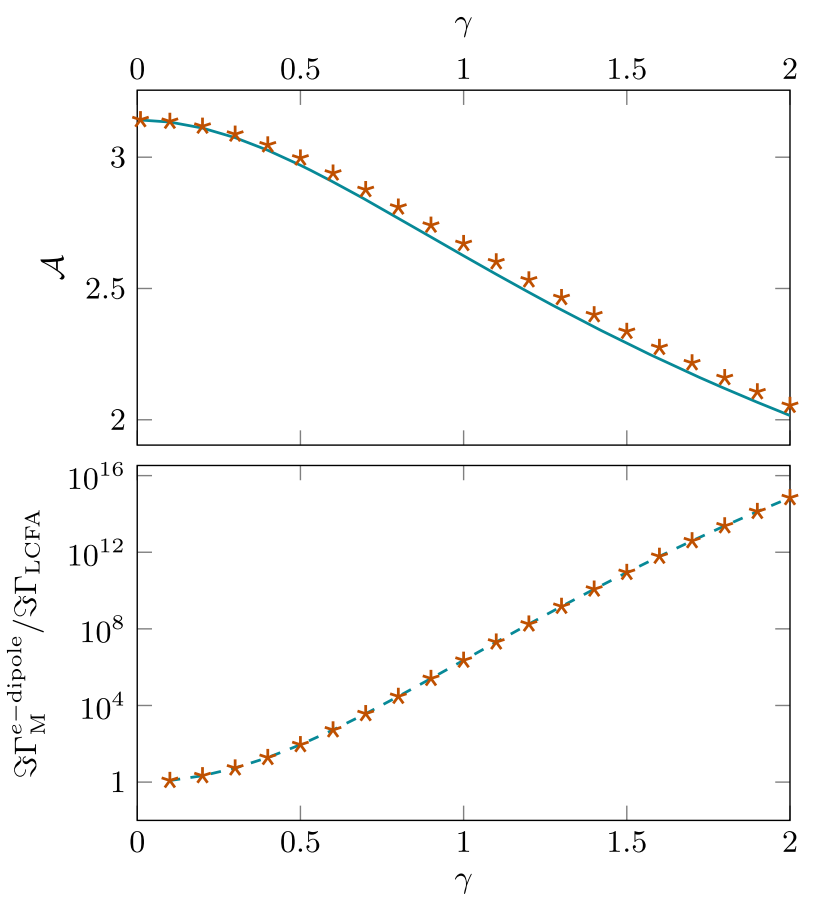

FIG. 12. Top: Instanton action for the Gaussian $e$-dipole given by (40) and (41) which depends on space and time (markers) in comparison to a spatially homogeneous electric field $\boldsymbol{E}=$ $E \exp \left\{-\omega^{2} t^{2}\right\} \boldsymbol{e}_{z}$ with Gaussian time dependence (solid curve). Bottom: Ratio of the effective action and the locally constant field approximation for $E=0.033 \mathrm{~m}^{2} / q$, with a dashed connecting line as a visual aid. 


$$
\Im \Gamma_{\mathrm{LCFA}}=\int \mathrm{d}^{4} x \frac{(q \mathcal{E}(x))^{2}}{(2 \pi)^{3}} \exp \left(-\pi \frac{m^{2}}{q \mathcal{E}(x)}\right)
$$

Here $\mathcal{E}$ denotes the Lorentz invariant electric field component of the field strength tensor $F_{\mu \nu}$ which is given by $\mathcal{E}=\sqrt{-F_{\mu \nu} F^{\mu \nu} / 2}$ for electrically dominated fields $F_{\mu \nu} F^{\mu \nu}<0$ and $\mathcal{E}=0$ for magnetically dominated fields $F_{\mu \nu} F^{\mu \nu}>0$. Again, for electric fields well below the critical field strength, the spacetime integral can be approximated using the saddle-point method, where the global maximum of $\mathcal{E}$ dominates. For the field profile given by (40) and (41), the global maximum lies at the origin where $\mathcal{E}(t=0, r=0)=E$ such that we obtain the same exponent as in (1). In order to derive the prefactor, we have to calculate the second spacetime derivatives $\partial_{\mu} \partial_{\nu} \mathcal{E}$ at the origin, which determine the effective four-volume for pair creation. Altogether, we find

$$
\Im \Gamma_{\mathrm{LCFA}}^{e-\text { dipole }} \approx \frac{5 \sqrt{5}}{2(2 \pi)^{3} \gamma^{4}} \exp \left(-\pi \frac{m^{2}}{q E}\right),
$$

where the factor $1 / \gamma^{4}$ corresponds to the effective fourvolume for pair creation. This result of the locally constant field approximation is shown in the bottom plot of Fig. 12 in comparison to the worldline instanton result.

As expected, the worldline instanton result tends to the locally constant field approximation for small values of $\gamma$, while it is exponentially larger for higher $\gamma$. For the parameters considered in [53] the adiabaticity is very small, with $\gamma<10^{-3}$, so the locally constant field approximation is accurate. For high frequency pulses however, the pair production rate is higher than the constant field estimate.

\section{G. Transversal standing wave}

Let us now briefly consider a purely transversal inhomogeneity. Two counterpropagating laser beams create a standing wave pattern, i.e., $\boldsymbol{E}=\cos (\omega t) \cos (k x) \boldsymbol{e}_{z}$ with $k=\omega$. In [10] the authors find that omitting the spatial inhomogeneity leads to qualitatively incorrect results in the high frequency regime. In [8] strong deviations in the momentum spectrum have been found in the homogeneous approximation as well.

Let us compare the total pair-creation probability for a standing wave $\boldsymbol{E}=\cos (\omega t) \cos (k x) \boldsymbol{e}_{z}$, where $k=\omega$ with that of a spatially homogeneous but time-dependent sinusoidal field $\boldsymbol{E}=\cos (\omega t) \boldsymbol{e}_{z}$. In the semiclassical regime $m^{2} \gg q E$ and for moderate values of $\gamma$ [55], we find that the two results agree quite well, see Fig. 13. It is easy to see that the transversal inhomogeneity does not change the instanton and thus the action [50], but the effect on the prefactor is not as obvious. Calculating the full effective action using the discrete instantons shows that while the prefactor does change, the difference from the homogeneous result is small and barely visible, see Fig. 13. Thus, omitting the spatial dependence is a fairly good

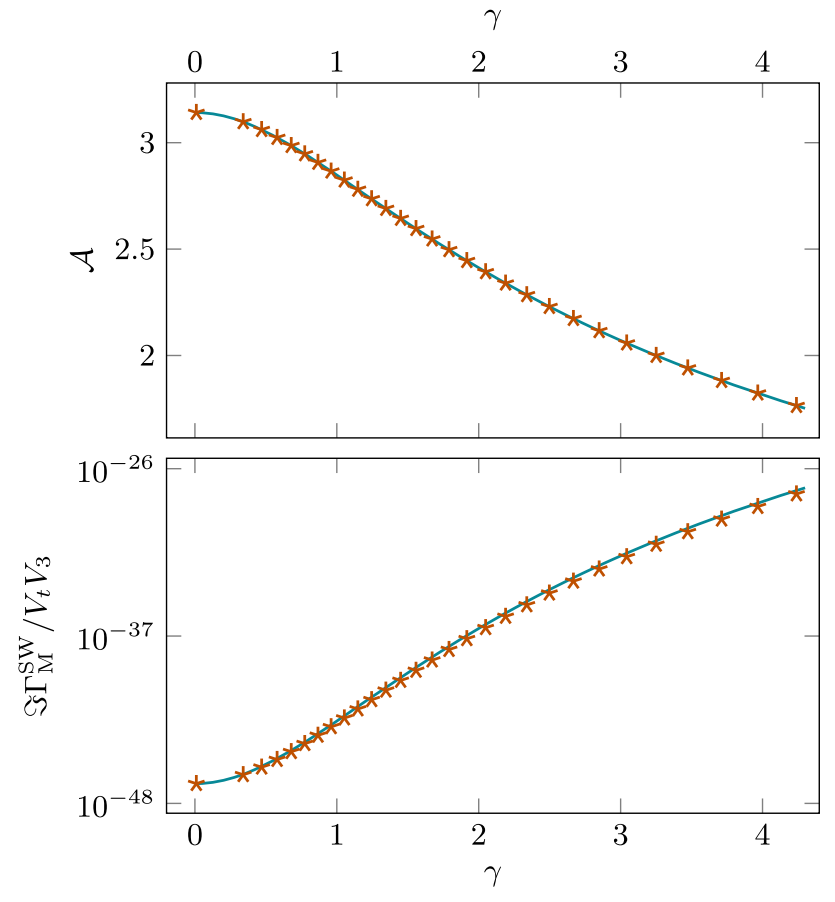

FIG. 13. Top: Instanton action for a transversally polarized standing wave (markers) compared to just the oscillating time dependent field (line). Bottom: The same comparison for the imaginary part of the effective action with $E=0.033 \mathrm{~m}^{2} / \mathrm{q}$ including the prefactor. The transversal inhomogeneity does not change the exponent at all, but has a small effect on the prefactor.

approximation in this regime. Note, however, that the momentum spectrum could still display noticeable differences between the standing wave and the purely time dependent field. See $[56,57]$ for a treatment of the spectrum in the worldline approach.

\section{H. Constant electric and magnetic fields}

In all examples up to now, the spin factor had only a small impact, apart from the trivial factor of 2 in the pair production probability. Let us finally consider a simple example where there is a large, qualitative difference between scalar and spinor QED, a parallel superposition of constant electric and magnetic fields of strength $E$ and $B$ respectively.

The (first term of the) effective action for this combination is given by (see e.g. [58] and references therein)

$$
\begin{aligned}
& \Gamma_{\text {Scalar }} \approx \frac{(q E)^{2}}{(2 \pi)^{3}} \pi \frac{B}{E} \operatorname{csch}\left(\pi \frac{B}{E}\right) \exp \left(-\pi \frac{m^{2}}{q E}\right), \\
& \Gamma_{\text {Spinor }} \approx \frac{2(q E)^{2}}{(2 \pi)^{3}} \pi \frac{B}{E} \operatorname{coth}\left(\pi \frac{B}{E}\right) \exp \left(-\pi \frac{m^{2}}{q E}\right) .
\end{aligned}
$$

Figure 14 depicts the prefactors of these expressions, so the $B / E$ dependence, together with the discrete instanton result, showing perfect agreement. 


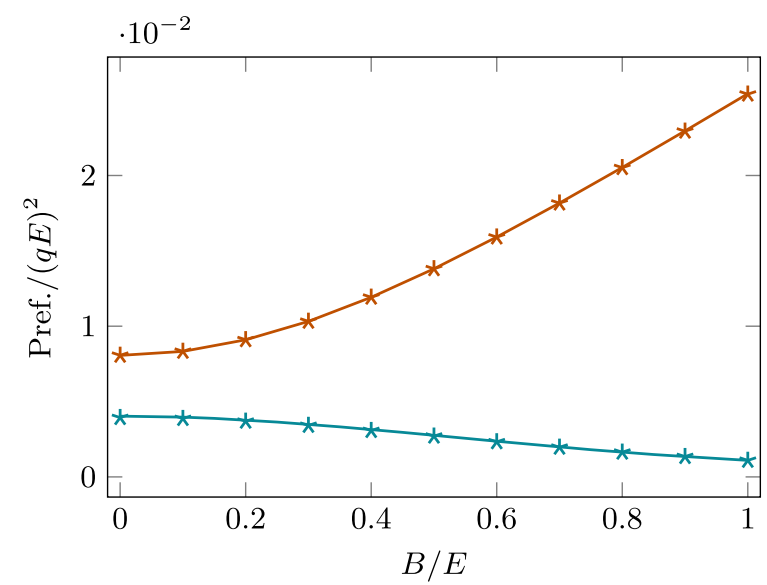

FIG. 14. Prefactor divided by $(q E)^{2}$ for the sum of a constant electric field and a magnetic field with varying $B / E$. The markers show the discrete instanton result, the lines the analytic expressions (46). The results for scalar QED are blue, the results for spinor QED red.

\section{SUMMARY AND CONCLUSION}

We have introduced a new approach to numerically implement the worldline instanton method for electronpositron pair creation. We use a discretization scheme that turns the infinite-dimensional path integral into a finite dimensional integration that we can then perform using Laplace's method. Crucially, this also means that the fluctuation prefactor is simply given by a finite dimensional determinant that can be computed without the great care that is needed for a properly normalized treatment of the functional determinant.

After having implemented the necessary root finding and continuation steps outlined in Secs. III, IV and V, full pair production results for arbitrary background fields can be obtained in minutes. Section VI gives a (by no means exhaustive) sample of such applications.

Although we used a frequency or inhomogeneity scale as the continuation parameter in all examples, we could have also chosen a different field parameter like the polarization direction or the ellipticity of the field, or even an entirely synthetic parameter to slowly transition to an especially complicated field configuration.

In this paper we have only considered cases for which there is one dominant instanton, which is continuously connected to a circular one in the constant field limit. It would be interesting for future studies to consider cases where there are more than one instanton, and where some of them might have a nontrivial topology.

\section{ACKNOWLEDGMENTS}

We thank Holger Gies and Christian Kohlfürst for interesting discussions. G. T. acknowledges support from the Alexander von Humboldt foundation.
[1] F. Sauter, Über das Verhalten eines Elektrons im homogenen elektrischen Feld nach der relativistischen Theorie Diracs, Z. Phys. 69, 742 (1931).

[2] W. Heisenberg and H. Euler, Folgerungen aus der Diracschen Theorie des Positrons, Z. Phys. 98, 714 (1936).

[3] F. Hund, Materieerzeugung im anschaulichen und im gequantelten Wellenbild der Materie, Z. Phys. 117, 1 (1941).

[4] J. Schwinger, On gauge invariance and vacuum polarization, Phys. Rev. 82, 664 (1951).

[5] T. D. Cohen and D. A. McGady, The Schwinger mechanism revisited, Phys. Rev. D 78, 036008 (2008).

[6] For example the extreme light infrastructure project, https:// eli-laser.eu/.

[7] C. Kohlfürst and R. Alkofer, On the effect of timedependent inhomogeneous magnetic fields in electronpositron pair production, Phys. Lett. B 756, 371 (2016).

[8] I. A. Aleksandrov, G. Plunien, and V. M. Shabaev, Momentum distribution of particles created in spacetime-dependent colliding laser pulses, Phys. Rev. D 96, 076006 (2017).

[9] C. Kohlfürst and R. Alkofer, Ponderomotive effects in multiphoton pair production, Phys. Rev. D 97, 036026 (2018).
[10] Q.Z. Lv, S. Dong, Y.T. Li, Z. M. Sheng, Q. Su, and R. Grobe, Role of the spatial inhomogeneity on the laser-induced vacuum decay, Phys. Rev. A 97, 022515 (2018).

[11] I. A. Aleksandrov, G. Plunien, and V. M. Shabaev, Dynamically assisted Schwinger effect beyond the spatially uniform-field approximation, Phys. Rev. D 97, 116001 (2018).

[12] C. Kohlfürst, Phase-space analysis of the Schwinger effect in inhomogeneous electromagnetic fields, Eur. Phys. J. Plus 133, 191 (2018).

[13] V. Fock, Die Eigenzeit in der klassischen und in der Quantenmechanik, Phys. Z. Sowjetunion 12, 404 (1937).

[14] Y. Nambu, The use of the proper time in quantum electrodynamics I, Prog. Theor. Phys. 5, 82 (1950).

[15] R. Feynman, Mathematical formulation of the quantum theory of electromagnetic interaction, Phys. Rev. 80, 440 (1950).

[16] R. Feynman, An operator calculus having applications in quantum electrodynamics, Phys. Rev. 84, 108 (1951).

[17] H. Gies and K. Langfeld, Quantum diffusion of magnetic fields in a numerical worldline approach, Nucl. Phys. B613, 353 (2001). 
[18] H. Gies, K. Langfeld, and L. Moyaerts, Casimir effect on the worldline, J. High Energy Phys. 06 (2003) 018.

[19] H. Gies and K. Klingmüller, Pair production in inhomogeneous fields, Phys. Rev. D 72, 065001 (2005).

[20] H. Gies and L. Roessler, Vacuum polarization tensor in inhomogeneous magnetic fields, Phys. Rev. D 84, 065035 (2011).

[21] I. K. Affleck, O. Alvarez, and N. S. Manton, Pair production at strong coupling in weak external fields, Nucl. Phys. B197, 509 (1982).

[22] G. V. Dunne and C. Schubert, Worldline instantons and pair production in inhomogenous fields, Phys. Rev. D 72, 105004 (2005).

[23] G. V. Dunne, Q. Wang, H. Gies, and C. Schubert, Worldline instantons and the fluctuation prefactor, Phys. Rev. D 73, 065028 (2006).

[24] A. Ilderton, G. Torgrimsson, and J. Wårdh, Nonperturbative pair production in interpolating fields, Phys. Rev. D 92, 065001 (2015).

[25] R. Schützhold, H. Gies, and G. V. Dunne, Dynamically Assisted Schwinger Mechanism, Phys. Rev. Lett. 101, 130404 (2008).

[26] H. Gies and G. Torgrimsson, Critical Schwinger Pair Production, Phys. Rev. Lett. 116, 090406 (2016).

[27] C. Schneider and R. Schützhold, Dynamically assisted Sauter-Schwinger effect in inhomogeneous electric fields, J. High Energy Phys. 1602 (2016) 164.

[28] G. V. Dunne and Q. Wang, Multidimensional worldline instantons, Phys. Rev. D 74, 065015 (2006).

[29] C. Schubert, Perturbative quantum field theory in the stringinspired formalism, Phys. Rep. 355, 73 (2001).

[30] C. Schubert, Lectures on the worldline formalism, School on spinning particles in quantum field theory: Worldline formalism, higher spins and conformal geometry, 2012, https://indico.cern.ch/event/206621/.

[31] H. Gies and J. Hämmerling, Geometry of spin-field coupling on the worldline, Phys. Rev. D 72, 065018 (2005).

[32] R. Feynman, Spacetime approach to nonrelativistic quantum mechanics, Rev. Mod. Phys. 20, 367 (1948).

[33] S. J. Rabello and C. Farina, Gauge invariance and the path integral, Phys. Rev. A 51, 2614 (1995).

[34] M. Stone, The Physics of Quantum Fields, Graduate Texts in Contemporary Physics (Springer, New York, 2000).

[35] B. Gaveau, E. Mihóková, M. Roncadelli, and L. S. Schulman, Path integral in a magnetic field using the Trotter product formula, Am. J. Phys. 72, 385 (2004).

[36] L.S. Schulman, Techniques and Applications of Path Integration (Dover Publications, New York, 2005).

[37] E. D'Hoker and D. G. Gagné, Worldline path integrals for fermions with scalar, pseudoscalar and vector couplings, Nucl. Phys. B467, 272 (1996).

[38] H. Gies and K. Langfeld, Loops and loop clouds-A numerical approach to the worldline formalism, Int. J. Mod. Phys. A 17, 966 (2002).

[39] L. D. Faddeev and V. N. Popov, Feynman diagrams for the Yang-Mills field, Phys. Lett. 25B, 29 (1967).

[40] J. Gordon and G. W. Semenoff, World-line instantons and the Schwinger effect as a Wentzel-Kramers-Brillouin exact path integral, J. Math. Phys. (N.Y.) 56, 022111 (2015).

[41] J. Gordon and G. W. Semenoff, Schwinger pair production: Explicit localization of the worldline instanton, arXiv:1612 .05909 .

[42] J. Zinn-Justin, Quantum Field Theory and Critical Phenomena, International Series of Monographs on Physics (Clarendon Press, Oxford, 1996).

[43] For the time-dependent fields, we found two negative eigenvalues, while for the time-independent fields, we found only one negative eigenvalue. In the latter case, this gives an additional factor of $i$ in the prefactor (the square root of the determinant of the Hessian), but this factor is canceled by the volume factor corresponding to the zero eigenvalue in time direction, after the transformation from Euclidean back to Minkowski time (as explained above). As expected from the comparison of scalar and spinor QED, the spin factor never changes its phase (but possibly its amplitude, see Fig. 14).

[44] E. L. Allgower and K. Georg, Introduction to Numerical Continuation Methods (Society for Industrial and Applied Mathematics, Philadelphia, 2003).

[45] W. C. Rheinboldt, Numerical continuation methods: A perspective, J. Comput. Appl. Math. 124, 229 (2000).

[46] O. Gould and A. Rajantie, Thermal Schwinger pair production at arbitrary coupling, Phys. Rev. D 96, 076002 (2017).

[47] D. F. Davidenko, On a new method of numerically integrating a system of nonlinear equations (Russian), Dokl. Akad. Nauk SSSR 88, 601 (1953).

[48] L. V. Keldysh, Ionization in the field of a strong electromagnetic wave, J. Exp. Theor. Phys. 20, 1307 (1965).

[49] G. Torgrimsson, C. Schneider, and R. Schützhold, SauterSchwinger pair creation dynamically assisted by a plane wave, Phys. Rev. D 97, 096004 (2018).

[50] M. F. Linder, C. Schneider, J. Sicking, N. Szpak, and R. Schützhold, Pulse shape dependence in the dynamically assisted Sauter-Schwinger effect, Phys. Rev. D 92, 085009 (2015).

[51] I. Gonoskov, A. Aiello, S. Heugel, and G. Leuchs, Dipole pulse theory: Maximizing the field amplitude from $4 \pi$ focused laser pulses, Phys. Rev. A 86, 053836 (2012).

[52] I. M. Bassett, Limit to concentration by focusing, Opt. Acta 33, 279 (1986).

[53] A. Gonoskov, I. Gonoskov, C. Harvey, A. Ilderton, A. Kim, M. Marklund, G. Mourou, and A. Sergeev, Probing Nonperturbative QED with Optimally Focused Laser Pulses, Phys. Rev. Lett. 111, 060404 (2013).

[54] M. A. Shifman, Wilson loop in vacuum fields, Nucl. Phys. B173, 13 (1980).

[55] Note that our semiclassical approach is based on the assumption that the expansion parameter $m^{2} /(q E)$ is sufficiently large and dominates over all other relevant parameters, such as $\gamma$. Relaxing this condition and considering field strengths $E$ closer to the Schwinger critical field can induce various deviations. For example, subdominant instanton solutions could yield non-negligible contributions (and lead to interference terms etc.) or other higher-order terms (of the large- $m$ expansion) can come into play. Note 
that this can also occur when $\gamma$ becomes so large that it spoils the role of $m^{2} /(q E)$ as the largest parameter.

[56] C. K. Dumlu and G. V. Dunne, Complex worldline instantons and quantum interference in vacuum pair production, Phys. Rev. D 84, 125023 (2011).
[57] Cesim K. Dumlu, Multidimensional quantum tunneling in the Schwinger effect, Phys. Rev. D 93, 065045 (2015).

[58] S. P. Kim and D. Page, Schwinger pair production in electric and magnetic fields, Phys. Rev. D 73, 065020 (2006). 